\title{
Interdisziplinarität als Schmerz-Löser
}

\section{Patienten erwarten Kompetenz in Diagnose und Therapie.}

Chronischer Schmerz trifft große Teile der Bevölkerung, die häufige Folge ist Arbeitsunfähigkeit, wie etwa von chronischen Rückenschmerzen. Interdisziplinäre Behandlungsansätze liefern die besten Ergebnisse.

„Schmerz hat sich mehr und mehr im sozialen Verständnis etabliert. Es kann heute sehr viel getan werden, um Menschen in das Sozial- und Arbeitsleben zu reintegrieren. Bei der Schmerzbehandlung ist nur ein interdisziplinärer Zugang sinnvoll, eine rein fachspezifische Schmerz-Behandlung erfasst das Problem nicht ausreichend ", umriss Univ.-Prof. Dr. Wilfried Ilias, Ehrenpräsident der Österreichischen Arbeitsgemeinschaft zur Schmerzbekämpfung \& Schmerzforschungszentrum ,Contra Dolorem' , die aktuelle Situation anlässlich des 3 . Wiener Schmerztags im vergangenen April.

Da Schmerz eine der häufigsten Ursachen ist, die Patienten zum Allgemeinmediziner führt, ist der Bedarf an adäquaten Behandlungskonzepten groß. Die Hälfte bis zwei Drittel der von chronischen Schmerzen betroffenen Menschen ist einem großen Leidensdruck ausgesetzt, der vielfach mit der Entwicklung psychischer Probleme korreliert. Die Schmerz-Diagnose erleben viele Patienten als sehr langwierig, oft vergehen Monate. „Die Behandlung chronischer Schmerzen ist kein punktuelles Ereignis. Schmerz-Behandlung besteht nicht nur aus einer Visite, sondern bedeutet eine auch langfristige Betreuung, bis man jenen $\mathrm{Zu}-$ stand erreicht hat, mit dem Schmerz-Patient und Behandler zufrieden sind", stellt Univ.-Prof. Dr. Sybille Kozek-Langenecker, Vorstand der Abteilung für Anästhesie und Intensivmedizin am Evangelischen Krankenhaus Wien fest.

\section{Multi- und interdisziplinärer Ansatz}

Behandlungskonzepte chronischer Schmerzen sind vielfach komplizierte Prozesse: Patienten, die über mehrere Jahre an quälenden Schmerzen leiden, sind verunsichert und/oder auch ängstlich. ,Contra dolorem' fordert „engagierte und empathische Netzwerke" für die Schmerz-Therapie ein, in denen Mediziner, Psychologen, Apothe- ker, Psychotherapeuten und Sozialpädagogen zusammenarbeiten sollen. Patienten erwarten zuvorderst eine rasche Linderung ihrer Schmerzen, erst dann können individuelle somato-psycho-soziale Konzepte zur Verbesserung der gesamten Lebenssituation schrittweise umgesetzt werden.

\section{Spezialfall kindlicher Schmerz}

Die Fortschritte in der SchmerzTherapie werden auch deutlich, wenn man sich die noch bis vor nicht allzu langer Zeit vertretene Ansicht verdeutlicht, dass Babys kein oder nur ein sehr eingeschränktes Schmerzempfinden hätten. „, Bei Babys und kleinsten Kindern sind wir mit einer mangelhaften Möglichkeit, den Schmerz zu artikulieren, konfrontiert", so KozekLangenecker zur speziellen Schmerz-Behandlung bei Kindern: „Im Spital kann man beispielsweise das kindliche Verhalten durch das Pflegepersonal beobachten lassen: Verändert sich durch die Therapien, die wir verabreichen, das Verhalten des Kindes so, dass wir es als Schmerz-Erleichterung interpretieren können? Prinzipiell steht uns ein gutes Repertoire an Medikamenten für die Akutbehandlung bei Kindern zur Verfügung. Die Medikamenten-Zulassungen für Kinder erreichen zwar nicht ganz den Umfang wie im Erwachsenen-Bereich, sodass man wissen muss, welche Strategien beim Einsatz bei Kindern unter 18 Jahren zugelassen sind. Wir haben aber genug im Köcher.“

\section{Contra Dolorem}

- unterstützt und berät (via Schmerztelefon) seit 2002 Schmerz-Patienten, betont den interdisziplinären Ansatz - wurde 2007 mit dem „Preis der Menschlichkeit" der Stadt Wien für soziales Engagement ausgezeichnet - veranstaltete im April den bereits, 3 . Wiener Schmerztag' im Rathaus für Interessierte, wobei auch die Fortbildungsveranstaltung (6 DFP) ,Schmerz interdisziplinär' angeboten wurde - hat einen ,Schmerzfragebogen' mit 12 Punkten konzipiert und aufgelegt, der dem behandelnden Arzt weitere Diagnostik und Therapieschritte erleichtern soll.

\section{Fazit}

Schmerz als komplexes Geschehen, das nicht nur die physiologische Komponente sondern sehr stark auch psychische und soziale Ebenen umfasst, muss in dieser Vielschichtigkeit optimal analysiert werden. Erst dann ist eine entsprechende Behandlung möglich. Zu bedenken ist jeweils auch die individuelle Reaktion auf die Therapie und bei Kindern, ebenso wie bei alten und dementen Menschen, die eingeschränkte Fähigkeit, Schmerz beziehungsweise Schmerzerleichterung verbal auszudrücken. Die genaue Beobachtung der Patienten ist hier von großer Bedeutung.

Informationen: www.schmerzinformation.org, EMail: info@schmerzinformation.org

\section{Buchempfehlung \\ SpringerWien NewYork

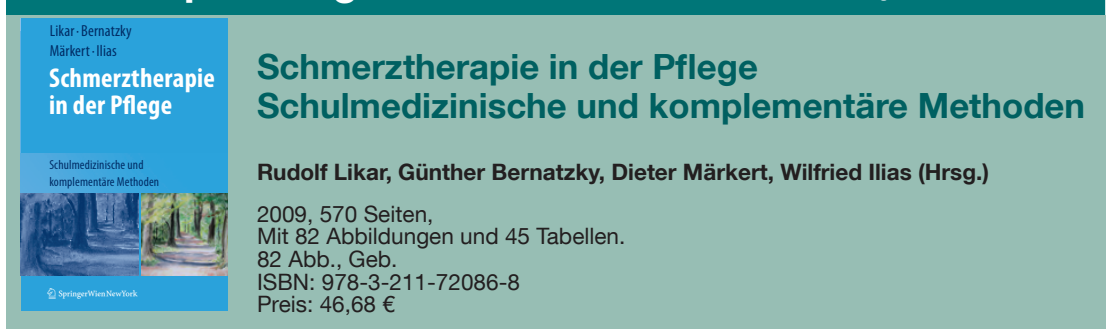

Aromapflege, Ayurveda, Entspannungsverfahren, Ergotherapie, Feldenkrais, Massage, Physiotherapie, Psychotherapie oder Wickel werden ausführlich anhand von zahlreichen praktischen Beispielen beschrieben. Ziel der Anwendung ist stets die Reduktion der Nebenwirkungen von Therapien. Die vorgestellten Methoden eignen sich aber auch zur Vorbeugung und können bei stagnierenden Heilungsprozessen erfolgreich eingesetzt werden. 